\title{
Depression in diabetes: Whom, When, and How to Screen
}

\author{
Maskey Robin ${ }^{1}$ \\ Additional Professor, Internal Medicine, BPKIHS \\ Dharan, Nepal
}

\section{INTRODUCTION}

Diabetes have a twofold increased risk of depression compared with the general population both sharing common biological and behavioral mechanisms. ${ }^{1}$ The two dominant hypotheses responsible for increased risk of depression in diabetic patients are as follows:

1) Due to biochemical changes directly due to the illness or its treatment and

2) The psychosocial demands or psychological factors related to the illness or its treatment.

The former hypothesis has also been referred to as a mood disorder due to a medical condition for which specific criteria have been formulated in Diagnostic and Statistical Manual of Mental Disorders. Both hypotheses are not mutually exclusive. ${ }^{2}$

Depression and diabetes results in poor glycemic control and self-management as well as increased health care costs and mortality. ${ }^{3}$ So, early recognition and treatment of depression and diabetes are essential for achieving optimal goals in the management of depression and in patients' overall quality of life.

\section{Whom to Screen?}

Depression is a condition in which patients meet at least five of the Diagnostic and Statistical Manual of Mental Disorder, Fifth Edition (DSM-5) criterion for major depressive disorder (MDD) nearly every day during the same 2-week period which are as follows: ${ }^{4}$

1 Depressed mood most of the day

2 Reduced interest or pleasure in all or almost all activities

Corresponding Address:: Maskey Robin1, Additional Professor, Internal Medicine, BPKIHS, Dharan, Nepal
3 Marked weight loss or weight gain

4 Sleep disorders

5 Psychomotor agitation or retardation

6 Fatigue or loss of energy

7 Feelings of worthlessness or guilt (excessive or inappropriate)

8 Problems concentrating or indecisiveness

9 Recurrent thoughts of death, suicidal ideation, or suicide attempt

\section{Screening for Depression and Diabetes Distress}

Experts suggest asking patients about alcohol use, since many people who are depressed self-medicate with alcohol, which also can affect glycemic control.

Because these depression scales are symptombased and do not link symptoms to a cause, experts believe that many people with diabetes who have depressive symptoms actually have emotional distress rather than clinical depression. ${ }^{5}$

Tools for screening and diagnosing depression include the 9-item Patient Health Questionnaire, ${ }^{6}$ Composite International Diagnostic Interview, ${ }^{7}$ Beck Depression Inventory, ${ }^{8}$ and the Center for Epidemiological Studies-Depression Scale. ${ }^{9}$

\section{When to screen?}

Patients with diabetes and depressive symptoms have mortality rates nearly twice as high as persons with diabetes and no depression. Depression may be due to biochemical changes of diabetes, like hyperglycemia, inflammation, activation of hypothalamic-pituitary-adrenal axis, or it could be due to the lifestyle factors associated with diabetes. ${ }^{10}$ 
Also, neurohormonal changes induced by depression, like hypercortisolism, can lead to insulin resistance and diabetes. Behavioral factors associated with depression, including lack of physical activity and poor diet, increase the risk of diabetes. All diabetics should be screened annually for depression. Two simple questions mentioned below help in screening the patients and it is clinically effective.

During the past month, have you often been bothered by feeling down, depressed, or hopeless?

During the past month, have you often been bothered by little interest or pleasure in doing things?

\section{How to screen?}

There are various instruments for how to screen depression in diabetes but the two-item case finding instrument proposed by Whooley et al., ${ }^{11}$ has sensitivity and specificity of $96 \%$ and $57 \%$, respectively, in identifying depression so it is widely used ${ }^{12}$ which includes two questions taken from the 27-item Primary Care Evaluation of Mental Disorders Procedure PRIME-MD ${ }^{12}$ :

These two questions are:

- During the past month, have you often been bothered by feeling down, depressed, or hopeless?

- During the past month, have you often been bothered by little interest or pleasure in doing things?

An answer of yes to either of these two questions needs a detailed evaluation for depressive disorder. The Whooley questions can be utilized as a simple, time-saving, validated instrument to screen for depression in diabetes care centers.

Another short, easy-to-administer instrument is the five-item World Health Organization Well Being
Index that assesses the psychological well-being. which includes five items that assess positive mood (good spirits, relaxation), vitality (being active and waking up fresh and rested), and general interests (being interested in things).

Each of the five items is rated on a 6-point Likert scale from 0 (=not present) to 5 (constantly present). A raw score less than 13 or an answer of 0 to 1 to any of the five items warrants detailed evaluation for depressive disorder.

That's why diabetes care professionals should incorporate the use of easy case-finding instruments, such as Whooley's questions and the WHO (Five) Well-Being Index in their routine practice.

Professional organizations across the globe should follow the example set by national guidelines in India, Germany, and other countries, which recommend regular assessment of psychological well being which is important part of diabetes care. ${ }^{13}$

\section{References:}

1. Holt RI, de Groot M, Lucki I, Hunter CM, Sartorius N, Golden SH. NIDDK international conference report on diabetes and depression: current understanding and future directions. Diabetes Care. 2014;37(8):2067-2077.

2. Talbot F, Nouwen A. A review of the relationship between depression and diabetes in adults: is there a link? Diabetes Care 2000;23(10):1556- 62.

3. Sumlin LL, Garcia TJ, Brown SA, et al. Depression and adherence to lifestyle changes in type 2 diabetes: a systematic review. Diabetes Educ. 2014 Jun 17. pii: 0145721714538925.

4. American Psychiatric Association. Diagnostic ad Statistical Manual of Mental Disorders, Fifth Edition. 2013.

5. Fisher L, Mullan JT, Arean P, Glasgow RE, Hessler D, Masharani U. Diabetes distress but not clinical depression or depressive symptoms 
is associated with glycemic control in both cross-sectional and longitudinal analyses. Diabetes Care. 2010;33(1):23-28.

6. Kroenke, K, Spitzer, RL, Williams, JBW: The PHQ-9: Validity of a Brief Depression Severity Measure. Journal of General Internal Medicine. 2001;16:606-613.

7. Wittchen HU. Reliability and validity studies of the WHO - Composite International Diagnostic Interview (CIDI): a critical review. J Psychiatr Res. 1994;28(1):57-84.

8. Beck AT, Ward CH, Mendelson M, Mock J, Erbaugh J, et al. An inventory for measuring depression. Arch Gen Psychiatry. 1961;4:5362.

9. Radloff LS. The CES-D scale: A self-report depression scale for research in the general population. Appl Psychol Meas. 1977;3:385401.

10. Raval A, Dhanaraj E, Bhansali A, Grover S, Tiwari P. Prevalence and determinants of depression in type 2 diabetes patients in a tertiary care centre. Indian J Med Res 2010;132:195-200.

11. Whooley MA, Avins AL, Miranda J, Browner WS. Case-finding instruments for depression. Two questions are as good as many. J Gen Intern Med 1997;12:439-45.

12. WHO. (Five) Well-Being Index (1998 version): Available from: http://cure4you.dk/354/WHO5_English.pdf [Last accessed on 2013 Mar 22]. 13. Kalra S, Sridhar G, Balhara YP, Sahay R, Bantwal $\mathrm{G}$, Baruah M, et al. National recommendations: Psychosocial management of diabetes in India. Indian J Endocrinol Metab 2013;17:376-9 